\title{
Design of Virtual Multimeter Based on VIIS-EM Platform
}

\author{
Zhengling $\mathrm{Wu}^{1}$, Haiyan $\mathrm{Wu}^{2}$ \\ ${ }^{1}$ College of information and control engineering, Jilin Institute of Chemical Technology, Jilin, Jilin \\ 132022, PR China; \\ ${ }^{2} 6354$ Research Institute of China Industrial Group Company, Jiujiang, Jiangxi 332000, PR China)
}

Key words: Virtual multimeter, LabVIEW, FPGA, USB

\begin{abstract}
From enriching VIIS-EW independently researched and developed by Jilin University, the development of virtual digital multimeter was finished for the purpose of designing modern intelligent digital multimeter. With the idea of modularization, the virtual digital multimeter was designed for the module with $3 \mathrm{U}$ card size, and the communication between controller was achieved using dual port RAM produced by FPGA. The main measuring circuit was composed of intelligent digital multimeter integrated circuit, to achieve the purpose of getting high performance objective through simple way. The instrument driver was designed by the form of the dynamic link library, and the I/O operations related with instrument were all encapsulated into function for the calling of application program. With the instrument operations the application calls for. The application program is developed by LabVIEW, and the communication with controller was realized through the CLF node. The Designed virtual digital multimeter did not only possess the basic measurement functions of traditional digital multimeter, but also had the advantages of data analysis, storage, report, network communication and so on, therefore, it can be applied to electrical and electronic measurements and other related fields.
\end{abstract}

\section{Introduction}

With the wide application of virtual instrument technology and the upgrade of computer software and hardware platform, the instrument develops to the direction of multi functions, high precision and integrated. Based on the advantages of virtual instrument technology, Virtual Instrument Laboratory of Jilin University Instrument Science and electrical engineering researched and developed a test system independently aimed at the forefront of international test, i.e. Virtual Instrument Integration System for Electronic Measuring (VIIS-EM). It is a set of modern measurement system which integrates signal source, signal frequency measurement, logic analysis, network application, and it can be widely used in industrial control, electronic measurement, experimental teaching and other related fields ${ }^{[1-2]}$.

Digital multimeter is one of the most widely used and most effective digital instrument in electronic measurement and maintenance and other related works. As a modern measurement system researched and developed independently, modular universal measuring instrument - virtual multimeter is essential.

Starting from enriching and perfecting the VIIS-EM platform, we design a VIIS-EM platform based on virtual multimeter for the purpose of designing modern intelligent digital multimeter in accordance with the mechanical specification and protocol of VIIS-EM platform. The design using virtual instrument technology combined with the powerful information processing ability of 
computer, and the function which a part of the hardware circuit couldn't achieve was realized by the software, making the investment of design smaller and update speed faster.

\section{Overall structure of VIIS-EM platform and system}

\section{VIIS-EM platform}

The overall structure of VIIS-EM system is shown in Figure 1. Based on the idea of modularization, the whole system adopted the way of "one controller + multi function modular instrument", and the communication between the upper computer and the host computer was realized through USB2.0. The controller and modular instrument were designed for 3U size (3.94 inch $x 6.3$ inch) and embedded in the chassis. There was a DC regulated power supply integrated in the chassis, which could provide $\pm 5 \mathrm{~V}, \pm 12 \mathrm{~V}$ DC voltage. At present, in the designed chassis there were 9 equipment slots and No. 0 slot was used for the controller slot, and the others were for the modular instrumentation slots ${ }^{[3-5]}$.

At present VIIS-EM platform developed by Jilin University has independently researched and developed, and the modular instruments which have put into experimental teaching application include digital storage oscilloscope, signal generator, LCR meter, logic analyzer, digital integrated circuit tester, tester for frequency response characteristic, frequency counter and programmable power supply. As a set of modern measurement system, it is imperative to research and develop digital multimeter.

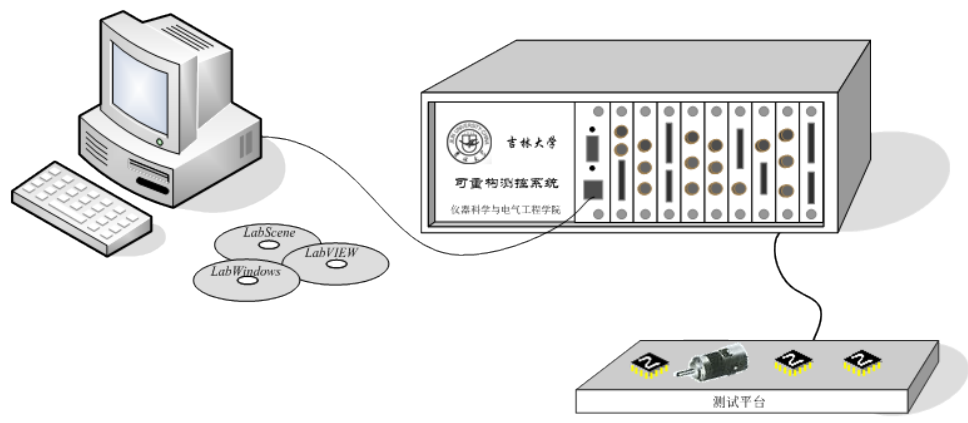

Figure 1 Overall structure of VIIS-EM system

\section{The overall structure of the virtual multimeter}

Combined with many advantages of virtual instrument technology, the designed virtual multimeter need possess these basic test functions such as DC voltage, AC voltage, DC current, alternating current, resistor, circuit on-off and diode test, and the specific requirements are as follows.

AC, DC voltage range: $400 \mathrm{mV}, 4 \mathrm{~V}, 40 \mathrm{~V}, 400 \mathrm{~V}$, accuracy: + (1\%);

AC, DC current range: $400 \mathrm{~mA}, 4 \mathrm{~A}$, accuracy: $+(2 \%)$

Resistance range: $400 \Omega, 4 \mathrm{k} \Omega, 40 \mathrm{k} \Omega, 4 \mathrm{M} \Omega, 40 \mathrm{M} \Omega$, accuracy: $+(1 \%)$

The design of virtual multimeter need display the measuring results in the digital, pointer, graphics and other forms at the same time; the measurement results such as mean value, extremum statistical analysis could be analyzed and processed; the range can achieve automatic switching, automatic adjusting to zero and calibration; it need possess good man-machine conversation ability and display the running status, work status and other information of equipment; the data can be observed for long time, and the display, storage and playback of measured data could be carried out; the network communication can be realized.

According to the VIIS-EM system structure and bus communication protocol, the design of modular virtual multimeter device board is mainly divided into two parts of software and hardware. 
The hardware part is composed of virtual multimeter hardware module and USB controller, and the software is composed of three parts: virtual multimeter driver, USB controller driver and application program. Figure 2 shows the overall structure of virtual digital multimeter, the virtual arrow in the figure represents a corresponding relationship between the information control, and the real arrow indicates the control flow.

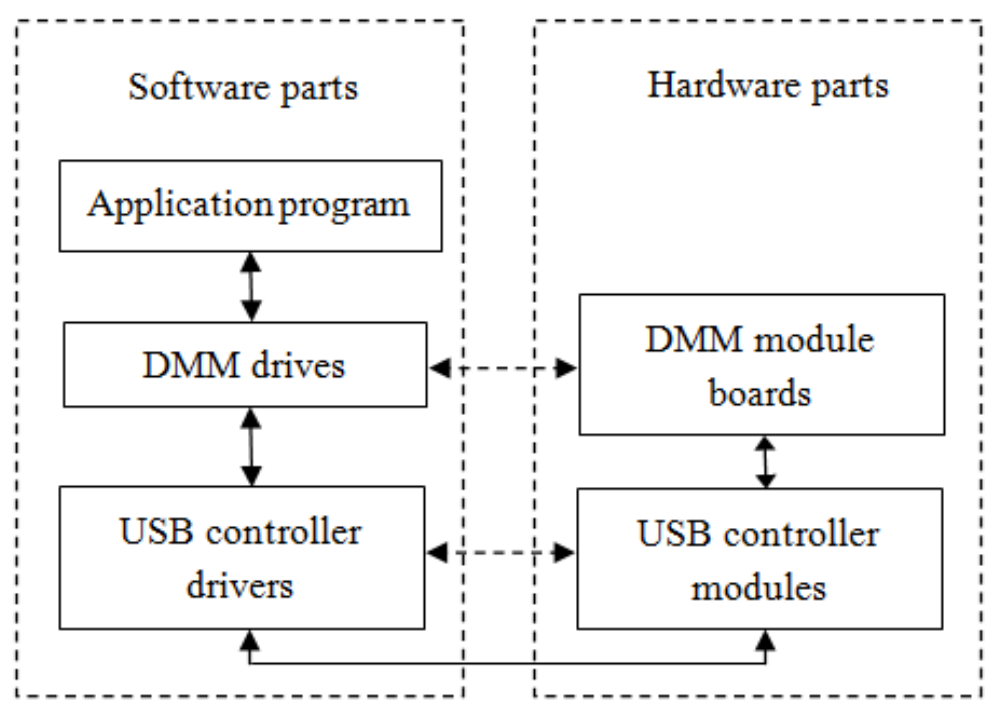

Figure 2 Overall structure of the virtual multimeter

\section{Design of system hardware}

\section{Overall structure of hardware}

According to the specific function, the virtual multimeter module board is mainly composed of three parts: measurement module, function conversion module, control and interface communication module, and the overall structure of module is shown in Figure 3. The measurement module realize the input signal measurement; function conversion module complete switching of the input signal attenuation and main measuring function; control and communication interface module composed of FPGA and MCU achieve control system and bus communication interface function.

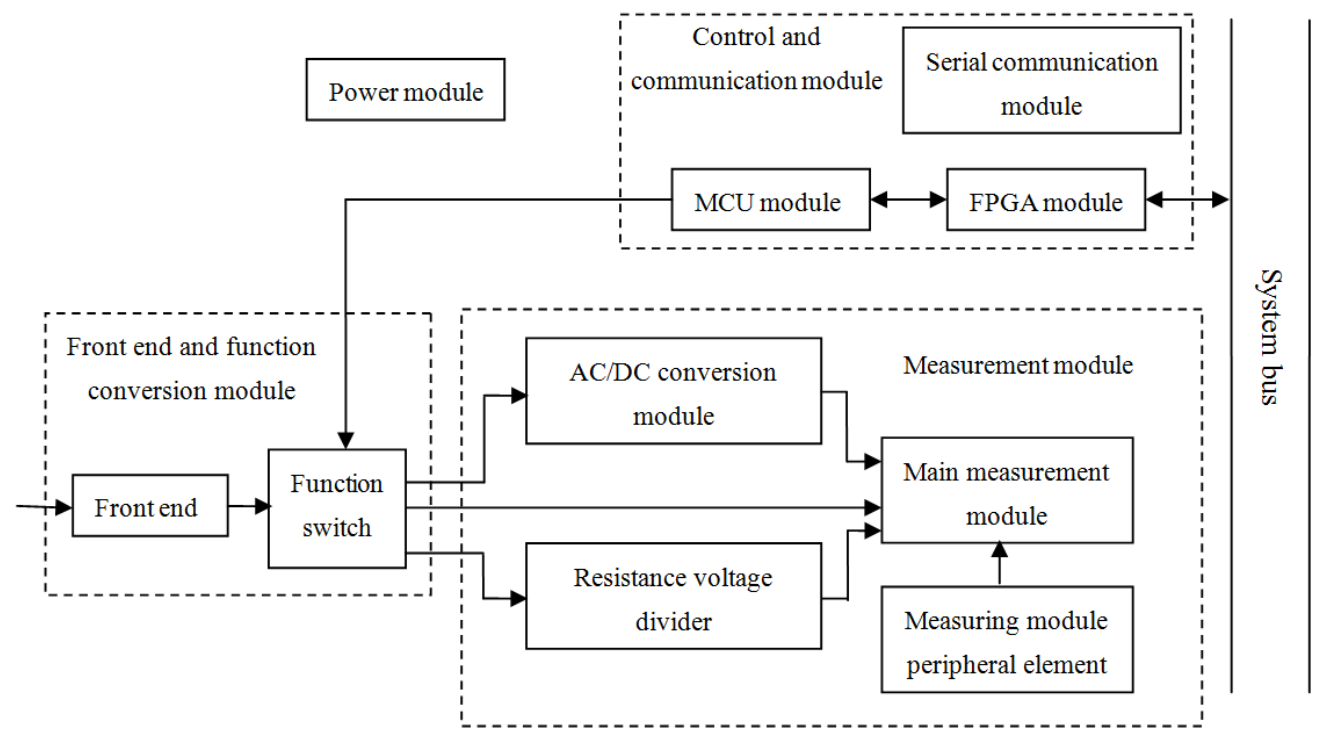

Figure 3 Hardware structure diagram of virtual digital multimeter 


\section{Main measuring circuit}

The measurement circuit is the core of virtual multimeter hardware part, and the single chip intelligent digital multimeter universal chip MAX134 is used to design. The integrated circuit has the advantages of high integration degree, few peripheral components, simple circuit, etc., and the development period is greatly saved. The maximum count of MAX134 usually take $\mathrm{N}= \pm 39999$; if the reserve position (D0) is specifically used for the microprocessor auto zero correction, $\mathrm{N}=$ \pm 3999 ; $N$ will increase to 79999 when the unipolar signal is measured. The count value increases 20 times higher than the general used A/D converter of 3 and a half. The main circuit of system is shown in Figure 4.

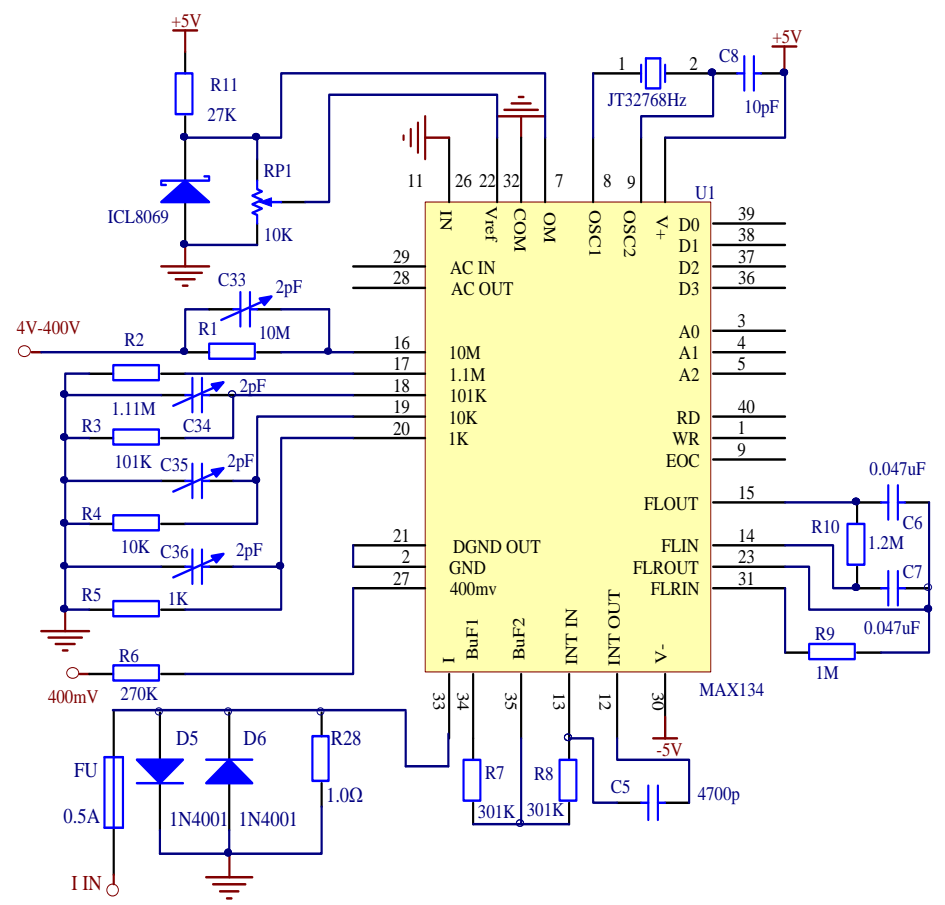

Figure 4 Circuit diagram of voltage measurement mode for virtual multimeter

According to the specific test function, the measuring circuit includes the current measurement mode, the voltage measurement mode, the resistance measurement mode and the diode measurement mode. Only 400mV range can be directly input when MAX134 measures voltage, and $4 \mathrm{~V}-4000 \mathrm{~V}$ range need be input through the voltage dividing network. R5 R2 is a voltage dividing network, C4 C7 is used for frequency compensation, and at the same time R1 R5 and C4 C7 constitute RC broadband non distortion attenuator.

33 pin input circuit of MAX134 is the current measurement mode input circuit, $\mathrm{R}_{28}$ take $1.0 \Omega$, $1 \mathrm{~W}$ when $400 \mathrm{~mA}$ measurement range; (for $4 \mathrm{~A}$ file, $\mathrm{R}=0.1 \Omega, 10 \mathrm{~W}$ ). The input side also increases the fuse of $0.5 \mathrm{~A}$ (for 4A file, FU takes 5A) as the overcurrent protection; D5 and D6 are used as overvoltage protection circuit.

Referenced voltage source ICL8069 provides reference voltage (1.2V) of resistance test, while ICL8069 get $655 \mathrm{mV}$ reference voltage $\mathrm{V}_{\mathrm{REF}}$ into the 26 feet of MAX134 through a precision potentiometer, which is used to suppress the power frequency interference. R7, R8, C5 in another circuit are the fringe integral elements of MAX134 internal integrator; R9, R10, C6 and C7 are the periphery filter elements in the filtering amplifying circuit. In the measurement of traffic flow, MAX134 also need connect external AC/DC conversion circuit.

MAX134 uses the proportional method to measure the resistance, and the input pin is the same as the voltage test, which is related to the function switching circuit. For circuit through the test, it is essentially measuring the resistance $R$ between two points, usually speaking, if $R<50 \Omega$, it is 
considered a short circuit between two points, from the program to the MAX134 control word is written, control buzzer sound.

\section{Function conversion circuit}

The function conversion circuit is composed of the relay, the precision resistance and so on, and the circuit diagram is shown in Figure 5. By controlling the switch of the relay, a controllable attenuation network was formed with the coordination of MAX134 internal analog switch, to achieve the switching between various test functions.

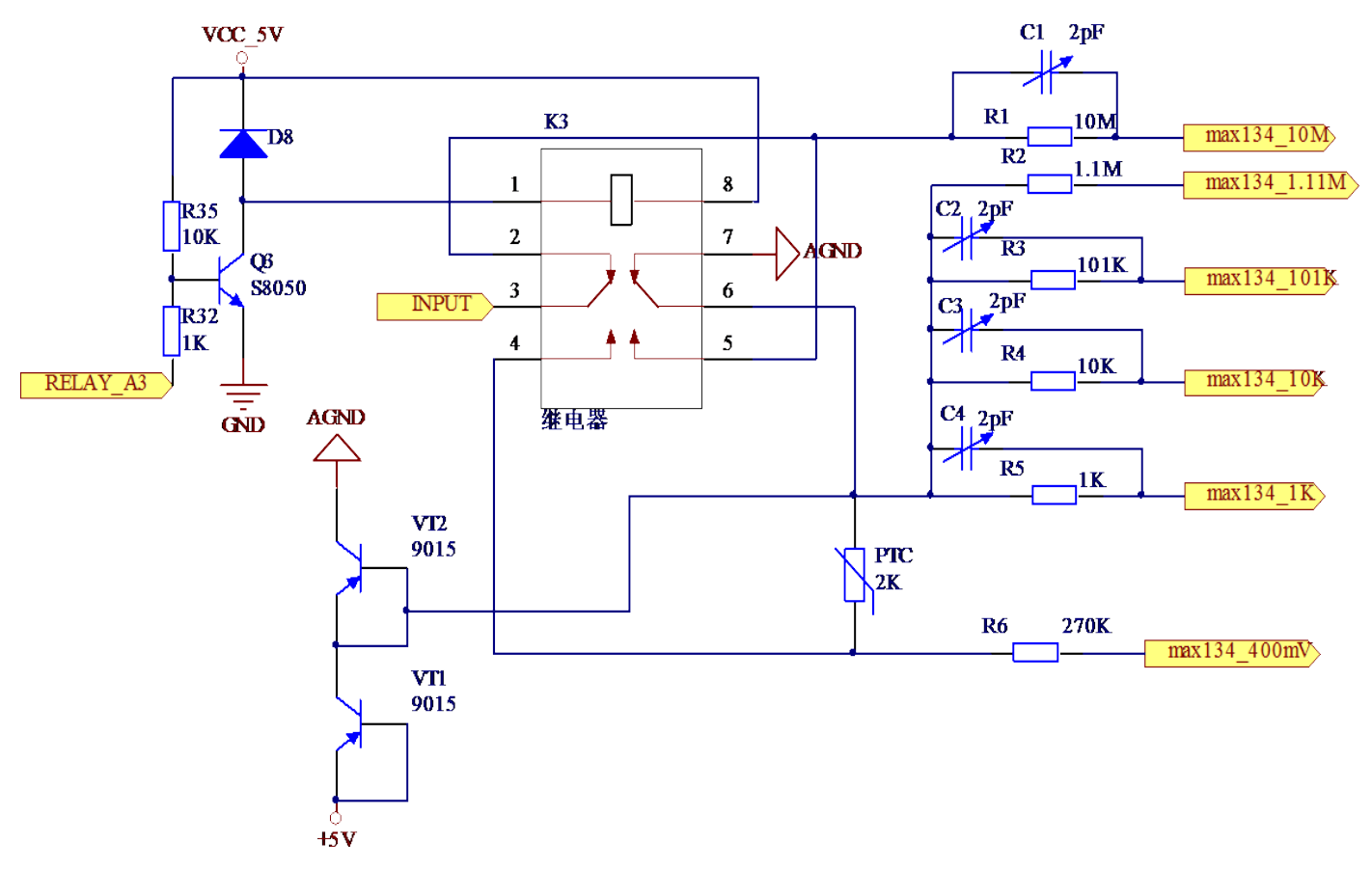

Figure 5 Function conversion circuit diagram

When the control signal RELAY_A3 is low level, the internal contacts of pin 2 and pin 3 in relay are connected; the internal contacts of pin 6 and pin 7 are connected. Matching with MAX134 internal analog switch (by writing to the MAX134 control word to achieve its control), it could make R1 R6 constitute voltage dividing resistance network, to form voltage testing circuit, so as to achieve voltage and diode test function.

When the control signal RELAY_A3 is high level, the internal contacts of pin 3 and pin 4 in relay are connected; the internal contacts of pin 6 and pin 5 are connected. Matching with the control of MAX134 internal analog switch, it could make R1 to R6 constitute reference resistor network, to form resistance test circuit, so as to realize the test function of resistance and circuit breaking.

\section{Design of platform bus interface communication}

Each equipment board in VIIS-EM platform has a processor to achieve the communication with USB controller, which involves the communication between multiple processors to solve the problem. The VIIS-EM system realizes the above problems by adding dual port RAM between the multiple processors. The communication between the card and the controller of virtual multimeter is designed by this structure.

The multi processor communication in VIIS-EM platform using $1 \mathrm{~K}$ bytes of dual port RAM is IDT7130, and IDT7130 has two interrupt output pins and interrupt arbitration circuits which are the difference from the general dual port RAM, so we can use IDT7130 interrupt to enable multi 
processors establish communication ${ }^{[3]}$. Dual port RAM-IDT7130 is realized with FPGA, and the arbitration model is shown in Figure 6.

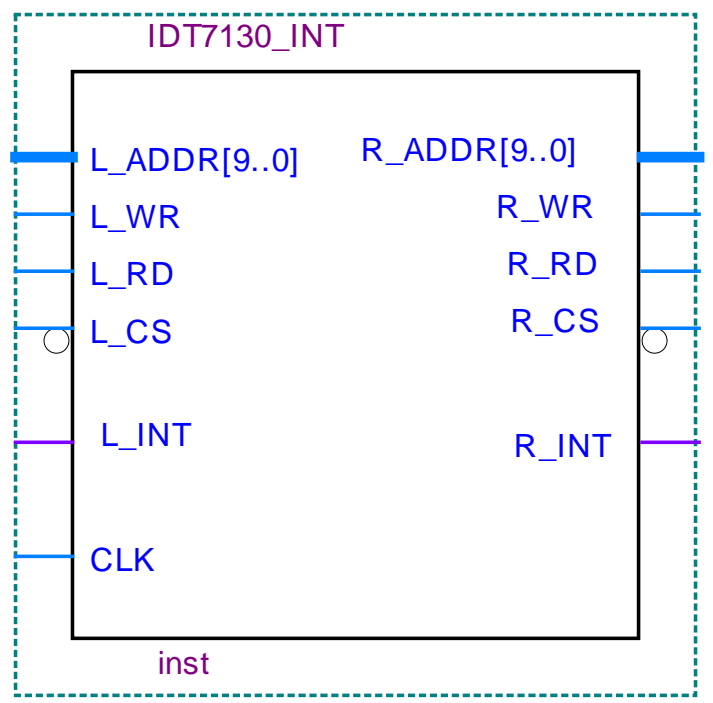

Figure 6 Interrupt arbitration model of IDT7130

\section{System software design}

Using the classic virtual instrument software architecture, the software part of the virtual multimeter is designed combined with the structural characteristics of the VIIS-EM platform, and the software structure is shown in Figure 2. Instrument driver is developed using VC6.0, and instrument panel control software is developed using LabVIEW.

The instrument software part mainly has two parts: the driver and the upper application program. For I/O driver design, the commands related to the hardware operation will be encapsulated into a function, to supply the program call in the form of dynamic link library. Therefore, the specific implementation details of instrument do not need to be known when preparing the application program, which greatly improves the efficiency and flexibility of programming. Users only need operate LabVIEW, to realize the communication with USB controller through the CLN function call dynamic link library, resulted in the program control to hardware module.

The LabVIEW program consists of three parts: the front panel, block diagram, icon/connection port. Front panel is used to simulate the real instrument; block diagram of the program controls the control object on the front panel using graphic language; icon/connection port is used to define the LabVIEW program as a subroutine to achieve modular programming.

\section{Test results}

Based on the function test at virtual multimeter VIIS-EM platform, the recorded data are shown as in table 2. In the test, resistance error for resistance test select $0.25 \%(1 / 4 \mathrm{~W})$; DC voltage source for DC voltage test is DF1731SL3A ( $\pm 20 \mathrm{~V}$, three significant digits); AC voltage test use function waveform generator DM32030 (1mV 10V, eight significant digits); when measuring current the resistance with $10 \Omega(0.25 \%, 1 / 4 \mathrm{~W})$ is connected with source voltage (DF1731SL3A) to output voltage, and the current value is measured using a Tektronix TDS2012B oscilloscope (100MHz, $1 \mathrm{GS} / \mathrm{s}$ ) measuring, to calibrate the system. Test results show that the design of virtual multimeter can meet the design requirements with the measured errors such aa resistance, current source, voltage source and so on. 
Table 1 Part function test data record of digital multimeter

\begin{tabular}{c|c|c|c|c}
\hline \multicolumn{2}{c|}{ Test item } & \multicolumn{2}{c}{ Test results } \\
\hline Test function & Test range & Nominal value & Measured value & Deviation \% \\
\hline \multirow{2}{*}{ Resistance measurement } & $400 \Omega$ & $300 \Omega$ & $299.8 \Omega$ & 0.067 \\
& $40 \mathrm{k} \Omega$ & $30 \mathrm{k} \Omega$ & $30.02 \mathrm{k} \Omega$ & 0.067 \\
& $40 \mathrm{M} \Omega$ & $20 \mathrm{M} \Omega$ & $20.05 \mathrm{M} \Omega$ & 0.250 \\
DC voltage measurement & $400 \mathrm{mV}$ & $200 \mathrm{mV}$ & $199.7 \mathrm{mV}$ & 0.150 \\
& $4 \mathrm{~V}$ & $3.00 \mathrm{~V}$ & $3.005 \mathrm{~V}$ & 0.167 \\
AC voltage measurement (100 Hz) & $40 \mathrm{~V}$ & $10.0 \mathrm{~V}$ & $10.03 \mathrm{~V}$ & 0.300 \\
DC current measurement & $400 \mathrm{~mA}$ & $200 \mathrm{~mA}$ & $199.5 \mathrm{mV}$ & 0.250 \\
\hline
\end{tabular}

Based on VIIS-EM platform, the function of the developed virtual multimeter is more powerful and more intelligent compared with the traditional multimeter. In addition to the traditional multimeter's basic functions, it can display measurement results with various forms; it can carry out software digital filter; it can process and analyse data, it can be used for data storage, display and playback; it can realize network communication, etc.

\section{Conclusions}

The virtual multimeter is developed based on the VIIS-EM platform, the results shows that its function and accuracy can meet the design requirements through repeated experiments. Using virtual instrument technology and combining the advantages of VIIS-EM platform, the designed virtual digital multimeter design can well compensate for the lack of traditional digital multimeter, save development cost, shorten the development cycle and maintain the high price advantage of digital multimeter. At the same time, it can increase the equipment module board for VIIS-EM platform, enrich and perfect the the test platform independent researched and developed, which makes the platform can be better applied to electronic measurement, industrial measurement and control, experimental teaching and other fields.

\section{References}

[1] Chaoping Yin, Bingren Zhang, Jixiang Zhao. Design of virtual digital integrated circuit tester based on VIIS-EW platform [J]. Application of Electronic Technique, 2013, 39(5): 89-92. (in Chinese)

[2] Jixiang Zhao, Bingren Zhang, Chaoping Yin. Design of virtual oscilloscope based on VIIS-EM [J]. Research and Exploration in Laboratory, 2013,32(10): 76-79. (in Chinese)

[3] Jianrong Wei. Research and design of reconfigurable measurement and control system [D]. Jilin University, 2006. (in Chinese)

[4] Zhaoxin Fan. Development of programmable power supply in virtual experiment teaching system [D]. Jilin University, 2008. (in Chinese)

[5] Yamin Mu, Ye Li, He Bai. Design of LCR testing instrument based on LabVIEW and FPGA [J]. Application of Electronic Technique, 2013, 39(11): 92-95. (in Chinese) 